\title{
ALGEBRAIC CYCLES AND THE HODGE STRUCTURE OF A KUGA FIBER VARIETY
}

\author{
B. BRENT GORDON
}

In memoriam-Michio Kuga

\begin{abstract}
Let $\tilde{A}$ denote a smooth compactification of the $k$-fold fiber product of the universal family $A^{1} \rightarrow M$ of elliptic curves with level $N$ structure. The purpose of this paper is to completely describe the algebraic cycles in and the Hodge structure of the Betti cohomology $H^{*}(\tilde{A}, \mathbb{Q})$ of $\tilde{A}$, for by doing so we are able (a) to verify both the usual and generalized Hodge conjectures for $\widetilde{A}$; (b) to describe both the kernel and the image of the Abel-Jacobi map from algebraic cycles algebraically equivalent to zero (modulo rational equivalence) into the Griffiths intermediate Jacobian; and (c) to verify Tate's conjecture concerning the algebraic cycles in the étale cohomology $H_{\mathrm{et}}^{*}\left(\tilde{A} \otimes \overline{\mathbb{Q}}, \mathbb{Q}_{l}\right)$. The methods used lead also to a complete description of the Hodge structure of the Betti cohomology $H^{*}\left(E^{k}, \mathbb{Q}\right)$ of the $k$-fold product of an elliptic curve $E$ without complex multiplication, and a verification of the generalized Hodge conjecture for $E^{k}$.
\end{abstract}

\section{INTRODUCTION}

Let $\tilde{A}$ denote a smooth compactification of the $k$-fold fiber product of the universal family $A^{1} \rightarrow M$ of elliptic curves with level $N$ structure. The purpose of this paper is to completely describe the algebraic cycles in and the Hodge structure of the Betti cohomology $H^{*}(\widetilde{A}, \mathbb{Q})$ of $\widetilde{A}$ : By doing so we are able (a) to verify both the usual and generalized Hodge conjectures [H, Gr1, Gr2] for $\widetilde{A} ;$ (b) to describe both the kernel and the image of the Abel-Jacobi map from algebraic cycles algebraically equivalent to zero (modulo rational equivalence) into the Griffiths intermediate Jacobian [Gf1, Gf2]; and (c) to verify Tate's conjecture [T] concerning the algebraic cycles in the étale cohomology $H_{\mathrm{et}}^{*}\left(\tilde{A} \otimes \overline{\mathbb{Q}}, \mathbb{Q}_{l}\right)$. En route to the main result we also completely describe the Hodge structure of the Betti cohomology $H^{*}\left(E^{k}, \mathbb{Q}\right)$ of the $k$-fold product of an elliptic curve $E$ without complex multiplication and deduce the validity of the generalized Hodge conjecture for $E^{k}$, for which the usual Hodge and Tate conjectures are already known [Mt1]. In $\S 1$ we recall the statements of these

Received by the editors April 13, 1990 and, in revised form, February 11, 1991.

1980 Mathematics Subject Classification (1985 Revision). Primary 14C30, 14 J40.

Key words and phrases. Kuga variety, elliptic curve, Hodge conjecture, generalized Hodge conjecture, Abel-Jacobi map, intermediate Jacobian, Tate conjecture, cusp forms.

This research was partially supported by the University of Oklahoma, the Vaughn Foundation and the National Science Foundation. 
conjectures and the construction of $\tilde{A}$, and state our results precisely.

In $\S 2$ we prove that $H^{m}\left(E^{k}, \mathbb{Q}\right)$ as a rational Hodge structure is the direct sum of a number of rational sub-Hodge structures each of which is the product of an irreducible rational Hodge structure of type $\{(\nu, 0),(\nu-1,1), \ldots$, $(0, \nu)\}$ with one of type $\{(\mu, \mu)\}$, where the product is actually realized by the cup product in $H^{*}\left(E^{k}, \mathbb{Q}\right)$, and $2 \mu+\nu=m$. The proof rests on the observations that the special Mumford-Tate group $\operatorname{SMT}(E)$ of $E$ [D4], called the Hodge group in [Mm1], is isomorphic to $\mathrm{SL}_{2}$, and that the (irreducible) $\operatorname{SMT}(E)$ submodules of $H^{m}\left(E^{k}, \mathbb{Q}\right)$ are precisely the (irreducible) rational subHodge structures. Thus the problem is reduced to determining the structure of $H^{m}\left(E^{k}, \mathbb{Q}\right)$ as an $\mathrm{SL}_{2}$-module, which is a classical sort of problem [W]. Once this is done, Theorem $\mathrm{A}$ and the generalized Hodge conjecture follow from the usual Hodge conjecture, which in the present case says that the one-dimensional $\mathrm{SL}_{2}$-invariants in $H^{m}\left(E^{k}, \mathbb{Q}\right)$ are algebraic [Mt1] (compare also $[\mathrm{K} 2, \S \mathrm{F}]$ ).

The proof of main Theorem $\mathrm{B}$, in $\S 3$, shows that there are three kinds of classes in $H^{*}(\widetilde{A}, \mathbb{Q})$ which generate this cohomology algebra over $\mathbb{Q}:$ (i) divisors in $\tilde{A}$ which are the closures in $\tilde{A}$ of divisors in a generic fiber $A_{\eta}$; (ii) the classes of algebraic cycles contained in the compactifying divisor at infinity; and (iii) classes which correspond to holomorphic cusp forms on $\Gamma(N)$, and their complex conjugates. The first two are of course algebraic, while the third live in rational Hodge structures of type $\{(\nu, 0),(0, \nu)\}$, for $1 \leq \nu \leq k+1$. To identify these classes is comparatively straightforward, the harder work is to show that they generate. Once this is done, however, we find that an especially strong form of the usual Hodge conjecture is true for $\widetilde{A}$, namely that all of $H^{d, d}(\widetilde{A})$ is spanned (over $\mathbb{C}$ ) by algebraic cycles. It is from this fact, and the main results of $p$-adic Hodge theory $[\mathrm{F}, \mathrm{FM}]$, that the Tate conjecture for $\widetilde{A}$ follows. A strong form of the generalized Hodge conjecture is also true for $\tilde{A}$ : The coniveau filtration is actually equal to the intersection of the Hodge filtration with the rational cohomology. Thus it is an easy matter to describe the (Griffiths) intermediate Jacobians of $\tilde{A}$ as well as the image therein of the Abel-Jacobi map applied to algebraic cycles on $\tilde{A}$ algebraically equivalent to zero, and it follows that Abel-Jacobi equivalence on those cycles is isogenous to incidence equivalence [Gf1, Gf2].

Both the present work and the previous work [Go2] which it complements were largely motivated by the work of Michio Kuga and his students and collaborators on algebraic cycles and the cohomology of group-theoretic families of abelian varieties, see [K1, K2, K3, HK, Ha, KS, KL]. Thus this paper is respectfully dedicated to the memory of Professor Kuga.

\section{Statement of Results}

A. Recall that a rational Hodge structure of weight $m$ is a finite-dimensional vector space $V$ over $\mathbb{Q}$ whose complexification $V(\mathbb{C}):=V \otimes_{\mathbb{Q}} \mathbb{C}$ has a Hodge decomposition $V(\mathbb{C})=\bigoplus_{0 \leq p \leq m} V^{p, m-p}$ with $V^{p, m-p}=\overline{V^{m-p, p}}$, or equivalently has a decreasing Hodge filtration $F_{H}^{r} V(\mathbb{C})$ with $F_{H}^{r} V(\mathbb{C}) \oplus \overline{F_{H}^{m+1-r} V(\mathbb{C})}=V(\mathbb{C})$ [D3]. These are related by $F_{H}^{r} V(\mathbb{C})=\bigoplus_{p \geq r} V^{p, m-p}$ and $V^{p, m-p}=F_{H}^{p} V(\mathbb{C}) \cap$ $\overline{F_{H}^{m-p} V(\mathbb{C})}$; in particular when $V$ has weight $2 d$, then $V^{d, d}=F_{H}^{d} V(\mathbb{C})=$ 
$F_{H}^{d} V(\mathbb{C}) \cap \overline{F_{H}^{d} V(\mathbb{C})}$. A rational Hodge structure $V$ is said to be of type $T$ iff $T$ is the set of pairs $(p, q)$ for which $V^{p, q} \neq\{0\}$. And an irreducible rational Hodge structure is, naturally, one which properly contains no nontrivial rational Hodge structures. The archtypical example of a rational Hodge structure of weight $m$ is the Betti (singular) cohomology $H^{m}(X, \mathbb{Q})$, where $X$ is a nonsingular complex projective variety.

For any such $X$, let $A H^{2 d}(X, \mathbb{Q})$ denote the space of algebraic cycles on $X$ (modulo homological equivalence), i.e. the subspace of $H^{2 d}(X, \mathbb{Q})$ spanned by classes Poincaré dual to closed subvarieties of codimension $d$ in $X$. Then $A H^{*}(X, \mathbb{Q}):=\bigoplus_{m} A H^{m}(X, \mathbb{Q})$ is a $\mathbb{Q}$-subalgebra of the cup product algebra $H^{*}(X, \mathbb{Q})$, where we let $A H^{m}(X, \mathbb{Q}):=\{0\}$ when $m$ is odd. Moreover it is not difficult to prove $[\mathrm{GH}]$ that

$$
A H^{2 d}(X, \mathbb{Q}) \subseteq H^{d, d}(X) \cap H^{2 d}(X, \mathbb{Q}) ;
$$

in particular this means that $A H^{*}(X, \mathbb{Q})$ consists of 1-dimensional rational Hodge structures, or Hodge cycles, as the elements of the right-hand side of (1.1) will be called. Conversely, the usual Hodge conjecture $[\mathrm{H}]$ asserts that (1.1) is in fact an equality, i.e. that every Hodge cycle is an algebraic cycle; see [So1] for further discussion of what is known about this conjecture.

B. The coniveau filtration $F_{C}^{r} H^{m}(X, \mathbb{Q})$, also called the codimension or arithmetic filtration, is the descending filtration defined by letting $F_{C}^{r} H^{m}(X, \mathbb{Q})$ be the subspace of $H^{m}(X, \mathbb{Q})$ of classes which vanish outside a Zariski-closed subset of $X$ of codimension $\geq r$ [Gr1, Gr2]. Equivalently,

$$
\begin{aligned}
F_{C}^{r} H^{m}(X, \mathbb{Q}) & =\sum_{Z} \operatorname{Im}\left[H_{Z}^{m}(X, \mathbb{Q}) \rightarrow H^{m}(X, \mathbb{Q})\right] \\
& =\sum_{q, W} \operatorname{Im}\left[H^{m-2 q}(W, \mathbb{Q}) \rightarrow H^{m}(X, \mathbb{Q})\right] \\
& =\bigcup_{U} \operatorname{ker}\left[H^{m}(X, \mathbb{Q}) \rightarrow H^{m}(U, \mathbb{Q})\right],
\end{aligned}
$$

where $Z$ runs over closed irreducible subschemes with $\operatorname{codim}_{X} Z \geq r$, and $W$ runs over nonsingular complex projective varieties of dimension $\operatorname{dim} X-q$ with a morphism $W \rightarrow X$ (inducing a Gysin map on cohomology), for $q \geq r$, and $U$ runs over Zariski-open subsets of $X$ with $\operatorname{codim}(X-U) \geq r$. The idea is that the $W$ 's may be taken to be resolutions of singularities of the $Z$ 's, and the $Z$ 's are the $(X-U)$ 's. The coniveau filtration may also be characterized as the abutment of the second spectral sequence, see [BO]. In any case it follows immediately that $F_{C}^{d} H^{2 d}(X, \mathbb{Q})=A H^{2 d}(X, \mathbb{Q})$. More generally $F_{C}^{r} H^{m}(X, \mathbb{Q})$ is a rational sub-Hodge structure of $H^{m}(X, \mathbb{Q})$, and

$$
F_{C}^{r} H^{m}(X, \mathbb{Q}) \subseteq F_{H}^{r} H^{m}(X, \mathbb{C}) \cap H^{m}(X, \mathbb{Q})
$$

[So1, Gr1, Gr2]. The generalized Hodge conjecture asserts that the largest rational Hodge structure contained in $F_{H}^{r} H^{m}(X, \mathbb{C}) \cap H^{m}(X, \mathbb{Q})$ is in fact $F_{C}^{r} H^{m}(X, \mathbb{Q})[\mathrm{Gr} 1, \mathrm{Gr} 2]$. An alternative, but equivalent formulation is that in addition to the usual Hodge conjecture, whenever $V(\mathbb{Q})$ is a simple sub-Hodge structure of $H^{m}(X, \mathbb{Q})$ then the greatest integer $r$ such that $F_{C}^{r} V(\mathbb{Q})=V(\mathbb{Q})$ equals the least integer $s$ such that $V^{s, m-s}(\mathbb{C}) \neq\{0\}$. The idea here is that the type of a rational sub-Hodge structure of $H^{m}(X, \mathbb{Q})$ should determine its level 
in the coniveau filtration. In fact, Hodge originally conjectured that equality should hold in (1.2) [H], but Grothendieck provided a counterexample to that and proposed these reformulations in [Gr1]; see the remark following Theorem 1 below.

C. Now let $E$ denote an elliptic curve (over $\mathbb{C}$ ) without complex multiplication, so that $\operatorname{End}(E)=\mathbb{Z}$, and let $E^{k}:=E \times \cdots \times E$ be the product of $E$ with itself $k$ times (over $\mathbb{C}$ ). Then the usual Hodge conjecture for $E^{k}$ may be proved by showing that there are no more Hodge cycles than those which are generated by (cup products of) classes of divisors [Mt1, Mt2]. Thus all the algebraic cycles on $E^{k}$ are linear combinations of the "obvious" ones of the form $\sigma_{*}\left(\Delta^{a} \times E^{b} \times O^{c}\right)$, where $\Delta \subset E \times E$ is the diagonal, $O \in E$ is a (base) point, and $\sigma$ permutes the $k$ factors of $E^{k}$. The following theorem incorporates this information and further describes the Hodge structure of $H^{*}\left(E^{k}, \mathbb{Q}\right)$.

Theorem A. Let $E$ be an elliptic curve without complex multiplication.

(i) $A H^{*}\left(E^{k}, \mathbb{Q}\right)$ is generated by divisor classes.

$$
H^{*}\left(E^{k}, \mathbb{Q}\right)=A H^{*}\left(E^{k}, \mathbb{Q}\right) \oplus \bigoplus_{\mu, \nu} A H^{2 \mu}\left(E^{k}, \mathbb{Q}\right) \cup V^{\nu}\left(E^{k}, \mathbb{Q}\right),
$$

(cup product) where $V^{\nu}\left(E^{k}, \mathbb{Q}\right) \subset H^{\nu}\left(E^{k}, \mathbb{Q}\right)$ is a direct sum of $\left(\begin{array}{l}k \\ \nu\end{array}\right)$ irreducible $(\nu+1)$-dimensional rational Hodge structures of type $\{(\nu, 0),(\nu-1,1)$, $\ldots,(0, \nu)\}$, and $0 \leq \mu \leq k$ and $1 \leq \nu \leq k$.

An immediate consequence is

Theorem 1. The generalized Hodge conjecture is true for $E^{k}$.

Proof. We have already observed that the usual Hodge conjecture holds for $E^{k}$. From Theorem A it follows that any simple rational Hodge structure, say $V \subset H^{m}\left(E^{k}, \mathbb{Q}\right)$, must be the product of an irreducible constituent of $V^{\nu}\left(E^{k}, \mathbb{Q}\right)$, for some $\nu$, with an algebraic cycle of Hodge type $(\mu, \mu)$, with $m=2 \mu+\nu$. But then $\mu$ is both the greatest integer such that $F_{C}^{\mu} V=V$ and the least integer such that $V^{\mu, m-\mu}(\mathbb{C}) \neq\{0\}$, as required.

Remark. These theorems provide examples of the generalized Hodge conjecture holding with strict inequality in (1.2), as well as examples where equality holds. For if 1 and $\tau$ generate the period lattice of $E$, then

$$
\operatorname{dim}_{\mathbb{Q}} F_{H}^{1} H^{k}\left(E^{k}, \mathbb{C}\right) \cap H^{k}\left(E^{k}, \mathbb{Q}\right)=\left(\begin{array}{c}
2 k \\
k
\end{array}\right)-\min \{\operatorname{deg} \tau, k+1\},
$$

where $\operatorname{deg} \tau=[\mathbb{Q}(\tau): \mathbb{Q}]$ if $\tau$ is algebraic or infinite if $\tau$ is transcendental [Grl, $\S 1]$. Since $F_{C}^{1} H^{k}\left(E^{k}, \mathbb{Q}\right)$ is a rational Hodge structure, and any rational Hodge structure of odd weight must have even dimension, equality in (1.2) must fail if $k$ is odd and $\operatorname{deg} \tau \leq k$ and odd. On the other hand, the methods of [Go2, §2] show that equality holds in (1.2), for $X=E^{k}$, whenever $\operatorname{deg} \tau \geq m$.

D. Now let $N \geq 3$ be fixed, let $M$ be the moduli space for elliptic curves with full level $N$ structure, let $\varphi^{1}: A^{1} \rightarrow M$ be the corresponding family of elliptic curves, and let $A:=A^{k}:=A^{1} \times_{M} \cdots \times_{M} A^{1}$ be the $k$-fold fiber product of $A^{1}$ with itself over $M$, with $\varphi:=\varphi^{k}: A \rightarrow M$. Although $M$ is not complete as an algebraic curve, there exists a unique nonsingular complete algebraic curve $\widetilde{M}$ such that $M^{\infty}:=\widetilde{M}-M$ is a finite set of points, or cusps. Furthermore, since 
$A^{1}$ is an elliptic surface over $M$, there is also a unique nonsingular complete relatively minimal model $\tilde{\varphi}^{1}: \widetilde{A}^{1} \rightarrow \widetilde{M}$ of $A^{1}$ [AMRT, Ko]. In fact, $\left(\tilde{\varphi}^{1}\right)^{-1}(p)$ is an $N$-gon of rational curves for $p \in M^{\infty}$, and $\widetilde{A}^{1}-A^{1}$ is the disjoint union over $p \in M^{\infty}$ of these.

If $k>1$ we may construct a smooth completion $\tilde{A}$ of $A$ by first letting $\bar{A}:=\widetilde{A}^{1} \times{ }_{\widetilde{M}} \cdots \times_{\widetilde{M}} \widetilde{A}^{1}$, with $\bar{\varphi}: \bar{A} \rightarrow \widetilde{M}$. Then there is a natural stratification of the singular locus of $\bar{A}$ : for each $p \in M^{\infty}$, the $j$-dimensional stratum of $\bar{\varphi}^{-1}(p)$ consists of $N^{k}\left(\begin{array}{l}k \\ j\end{array}\right)$ components each of which is a direct product of $j$ rational curves (and $(k-j)$ double points) of $\left(\tilde{\varphi}^{1}\right)^{-1}(p)$. By blowing up each component of each stratum, starting with the 0-dimensional and continuing through the $(k-1)$-dimensional, we get the nonsingular $\tilde{A}[\check{\mathrm{S}} 1]$. Another description of the nonsingular completion $\tilde{A}$ of $A$ is given in [D1, $\S 5$ ], and the methods of [AMRT] may be used as well. Letting $A^{\infty}:=\tilde{A}-A$ and $\varphi^{\infty}: A^{\infty} \rightarrow M^{\infty}$, we summarize the notation in the following diagram.

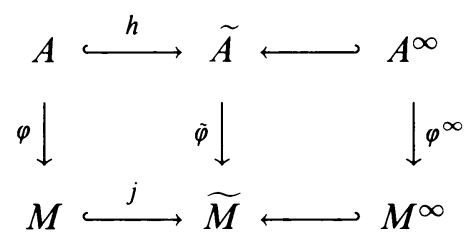

Note that $A$ and $\tilde{A}$ are families of Hodge type, in the terminology of [Mm2]. Theorem B. Let $\tilde{\varphi}: \widetilde{A} \rightarrow \widetilde{M}$ be as above, let $\eta$ denote a generic point of $\widetilde{M}$, and let $A_{\eta}:=\tilde{\varphi}^{-1}(\eta)$.

(i) $A H^{*}(\tilde{A}, \mathbb{Q})$ is generated by

(a) The classes of the closures in $\tilde{A}$ of divisors in $A_{\eta}$, and

(b) The classes of algebraic cycles supported on $A^{\infty}$.

$$
H^{*}(\tilde{A}, \mathbb{Q})=A H^{*}(\tilde{A}, \mathbb{Q}) \oplus \bigoplus_{\mu, \nu} A H^{2 \mu}(\tilde{A}, \mathbb{Q}) \cup W^{\nu}(\tilde{A}, \mathbb{Q}),
$$

(cup product) where $W^{\nu}(\tilde{A}, \mathbb{Q}) \subset H^{\nu}(\tilde{A}, \mathbb{Q})$ is a direct sum of irreducible rational Hodge structures of type $\{(\nu, 0),(0, \nu)\}$, and

$$
\operatorname{dim}_{\mathbb{Q}} W^{\nu}(\tilde{A}, \mathbb{Q})=\frac{1}{12}\left(\begin{array}{c}
k \\
\nu-1
\end{array}\right)(\nu N+6) N^{2} \prod_{p \mid N}\left(1-p^{-2}\right),
$$

with $0 \leq \mu \leq k+1$ and $1 \leq \nu \leq k+1$.

Remarks. 1. Since $A_{\eta}$ is isomorphic to the $k$-fold self-product of an elliptic curve without complex multiplication, Theorem A implies that the divisors generate all the algebraic cycles on $A_{\eta}$. Thus part (i) of this theorem identifies all the algebraic cycles on $\tilde{A}$ as linear combinations of the closures in $\tilde{A}$ of intersections of divisors on $A_{\eta}$ and cycles supported on $A^{\infty}$.

2. The formula for $\operatorname{dim}_{\mathbb{Q}} W^{\nu}(\tilde{A}, \mathbb{Q})$ may be written more simply as

$$
\operatorname{dim}_{\mathbb{Q}} W^{\nu}(\tilde{A}, \mathbb{Q})=2\left(\begin{array}{c}
k \\
\nu-1
\end{array}\right) \operatorname{dim} S_{\nu+1}(\Gamma(N)),
$$


where $S_{\nu+1}(\Gamma(N))$ is the space of holomorphic cusp forms of weight $\nu+1$ on the principal congruence subgroup $\Gamma(N) \subset \mathrm{SL}_{2}(\mathbb{Z})$ [Sm1, Sb]; see Lemma 5 below, and compare with [KS, K1, Š $1, \check{S} 2, S m 2]$ and $[Z, \S 12])$.

3. Theorem B and its consequences below were known to Shioda [So2] for $k=1$. This theorem may also be compared with the results in the analogous "compact case" studied in [HK, Go2].

The first consequence we draw from Theorem B is a strong form of the usual Hodge conjecture, which is valid for projective spaces, Grassmannians and flag varieties, but only rarely otherwise (see the comment in [HK]).

Theorem 2. $H^{d, d}(\tilde{A})=A H^{2 d}(\tilde{A}, \mathbb{Q}) \otimes \mathbb{C}$.

Proof. It follows immediately from part (ii) that there are no cohomology classes of type $(d, d)$ other than the algebraic cycles, even in $H^{*}(\widetilde{A}, \mathbb{C})$.

A strong form of the generalized Hodge conjecture is also true.

Theorem 3. $F_{C}^{r} H^{m}(\tilde{A}, \mathbb{Q})=F_{H}^{r} H^{m}(\tilde{A}, \mathbb{C}) \cap H^{m}(\tilde{A}, \mathbb{Q})$.

Proof. By Theorem B, every simple rational Hodge structure is the product of one of type $\{(\nu, 0),(0, \nu)\}$ with one of type $\{(\mu, \mu)\}$, where is the latter is algebraic. Such a sub-Hodge structure is contained in $F_{C}^{r} H^{m}(\widetilde{A}, \mathbb{Q})$ when $m=2 \mu+\nu$ and $r \leq \mu$, which are also precisely the conditions under which it is contained in $F_{H}^{r} H^{m}(\widetilde{A}, \mathbb{C})$.

E. With the detailed knowledge about the Hodge structure of $H^{*}(\tilde{A}, \mathbb{Q})$ which Theorem B provides, we can answer some of the questions posed by Griffiths [Gf2] about the intermediate Jacobians of $\widetilde{A}$ and the image and the kernel of the Abel-Jacobi map applied to cycles algebraically equivalent to zero. Recall [Gf1, Gf2, Mr] that the $r$ th (Griffiths) intermediate Jacobian is the complex torus

$$
\begin{aligned}
J^{r}(\tilde{A}) & :=F_{H}^{r} H^{2 r-1}(\tilde{A}, \mathbb{C}) \backslash H^{2 r-1}(\tilde{A}, \mathbb{C}) / H^{2 r-1}(\tilde{A}, \mathbb{Z}) \\
& \cong F_{H}^{k-r+2} H^{2 k-2 r+3}(\tilde{A}, \mathbb{C})^{*} / H_{2 k-2 r+3}(\tilde{A}, \mathbb{Z}),
\end{aligned}
$$

where it should to be understood that the quotient is actually by the image of $H^{2 r-1}(\widetilde{A}, \mathbb{Z})\left(\operatorname{resp} . H_{2 k-2 r+3}(\widetilde{A}, \mathbb{Z})\right)$ in $H^{2 r-1}(\widetilde{A}, \mathbb{C})\left(\operatorname{resp} . H^{2 k-2 r+3}(\widetilde{A}, \mathbb{C})^{*}\right.$, the dual of $\left.H^{2 k-2 r+3}(\tilde{A}, \mathbb{C})\right)$; the canonical isomorphism is a consequence of Poincare duality. In the language of [Gf2], the (total) Jacobian variety $J(\widetilde{A}):=$ $\bigoplus_{1 \leq r \leq k+1} J^{r}(\widetilde{A})$ is a graded self-dual complex torus, meaning that $J^{r}(\widetilde{A})$ is isogenous to the dual of $J^{k+2-r}(\widetilde{A})$ for $1 \leq r \leq k+1$. Let $\mathscr{A}^{r}(\widetilde{A})$ denote the group of algebraic cycles on $\tilde{A}$ of codimension $r$ which are algebraically equivalent to zero, modulo rational equivalence. Then an element $Z$ of $\mathscr{A}^{r}(\widetilde{A})$ is homologous to zero, and therefore may be interpreted as the boundary of a $(2 k-2 r+3)$-chain $C$. Thus the Abel-Jacobi map $\psi^{r}: \mathscr{A}^{r}(\widetilde{A}) \rightarrow J^{r}(\widetilde{A})$ is defined by mapping $Z$ to $\int_{C} \omega\left(\bmod H^{2 r-1}(\widetilde{A}, \mathbb{Z})\right)$ as $\omega$ runs over a basis for $F_{H}^{k-r+2} H^{2 k-2 r+3}(\widetilde{A}, \mathbb{C})$. The image $J_{\text {alg }}^{r}(\widetilde{A})$ of $\psi^{r}$ is known to be an abelian variety, while the elements of the kernel $\mathscr{A}_{\mathrm{AJ}}^{r}(\widetilde{A})$ of $\psi^{r}$ are said to be $A b e l$ Jacobi equivalent to zero. Let $J_{\mathrm{alg}}(\widetilde{A}):=\bigoplus_{1 \leq r \leq k+1} J_{\mathrm{alg}}^{r}(\widetilde{A})$. 
As an aid to studying Abel-Jacobi equivalence, Griffiths has defined the notion of incidence equivalence [Gf1, Gf2]: A cycle $Z$ (or its class) in $\mathscr{A}^{r}(\widetilde{A})$ is said to be incidence equivalent to zero iff for all smooth projective varieties $S$ and all algebraic cycles $Y$ of codimension $(2 k+2-r)$ on $S \times \widetilde{A}$ (modulo rational equivalence) the projection onto $S$ of the intersection product $((S \times Z) \cdot Y)$ is linearly equivalent to zero on $S$. Let $\mathscr{A}_{\text {inc }}^{r}(\widetilde{A})$ denote the subgroup of $\mathscr{A}^{r}(\widetilde{A})$ of classes incidence equivalent to zero. Then

$$
\mathscr{A}_{\mathrm{AJ}}^{r}(\tilde{A}) \subseteq \mathscr{A}_{\text {inc }}^{r}(\tilde{A})
$$

[Gf1], and it follows [Gf2] that there is a surjective homomorphism of abelian varieties $\lambda^{r}$ from $J_{\mathrm{alg}}^{r}(\widetilde{A})$ to the $r$ th Saito-Picard variety

Let $\operatorname{SPic}(\widetilde{A}):=\bigoplus_{1 \leq r \leq k+1} \operatorname{SPic}^{r}(\widetilde{A})$.

$$
\operatorname{SPic}^{r}(\widetilde{A}):=\mathscr{A}^{r}(\widetilde{A}) / \mathscr{A}_{\text {inc }}^{r}(\tilde{A}) .
$$

Referring to the notation of Theorem $\mathrm{B}(\mathrm{ii})$, let

$$
W^{0, \nu}(\widetilde{A}):=W^{\nu}(\tilde{A}, \mathbb{C}) \cap H^{0, \nu}(\widetilde{A})
$$

and $W^{\nu}(\tilde{A}, \mathbb{Z}):=W^{\nu}(\tilde{A}, \mathbb{Q}) \cap H^{\nu}(\tilde{A}, \mathbb{Z})$. Then we can describe the image and the kernel of the Abel-Jacobi map as follows.

Theorem 4. With the notations as above

(i) $J_{\text {alg }}^{r}(\widetilde{A})=W^{0,2 r-1}(\widetilde{A}) / W^{2 r-1}(\widetilde{A}, \mathbb{Z})$.

(ii) $\lambda^{r}: J_{\mathrm{alg}}^{r}(\tilde{A}) \rightarrow \operatorname{SPic}^{r}(\tilde{A})$ is an isogeny; i.e., Abel-Jacobi equivalence differs at most by an isogeny from incidence equivalence on $\tilde{A}$.

(iii) $J_{\mathrm{alg}}(\widetilde{A})$ and $\mathrm{SPic}(\widetilde{A})$ are both self-dual graded abelian varieties.

(iv) $J^{r}(\tilde{A})$ is isogenous to $\bigoplus_{\nu} \operatorname{dim}\left(A H^{2 r-2 \nu}(\tilde{A}, \mathbb{Q})\right) J_{\mathrm{alg}}^{\nu}(\tilde{A})$, with $1 \leq \nu \leq r$, where $m J_{\mathrm{alg}}^{\nu}(\tilde{A})$ signifies the sum of $m$ copies of $J_{\mathrm{alg}}^{r}(\widetilde{A})$; in particular, $J(\tilde{A})$ is a self-dual graded abelian variety.

Proof. Since sum of the tangent space at the origin of $J_{\mathrm{alg}}^{r}(\tilde{A})$ and its complex conjugate is exactly $F_{C}^{r-1} H^{2 r-1}(\tilde{A}, \mathbb{Q}) \otimes \mathbb{C}[\mathrm{Mr}, 4.3]$, part (i) is a consequence of Theorem 3. Then in [Gf $1, \S 10]$ it is shown that the generalized Hodge conjecture implies both that $\lambda^{r}$ is an isogeny and that $J_{\text {alg }}(\widetilde{A})$ is self-dual, though the self-duality follows from part (i) as well. This proves parts (ii) and (iii); then part (iv) follows directly from Theorem B(ii).

Remark. In the notation of Remark 2 following Theorem B, there is a natural isomorphism

$$
W^{2 r-1}(\tilde{A}, \mathbb{C}) \simeq\left(\begin{array}{c}
k \\
2 r-2
\end{array}\right)\left(S_{2 r}(\Gamma(N)) \oplus \overline{S_{2 r}(\Gamma(N))}\right) ;
$$

see Lemma 5 below, and compare [Z, 12.4]. Moreover, Shimura [Sm2, V] has shown that the quotient of $S_{2 r}(\Gamma(N))$ by the lattice obtained from $W^{2 r-1}(\widetilde{A}, \mathbb{Z})$ via this isomorphism is a complex torus which can be give the structure of an abelian variety. Thus $J_{\text {alg }}^{r}(\widetilde{A})$ and $J_{\text {alg }}(\widetilde{A})$ and $J(\widetilde{A})$ are isogenous to sums of these Shimura abelian varieties. This result may be compared with [KL], where the Weil intermediate Jacobians of the compact analogue of $\tilde{A}$ are also shown to be sums of these Shimura abelian varieties but with a modified complex structure. 
F. Another consequence of Theorem B, or more accurately, Theorem 2 , is that one of Tate's conjectures [T] is true for $\widetilde{A}$, as we now explain. To begin with, it is a consequence of the theory of canonical models [Sm3, D2], for example, that $M$, and therefore also $A$ and $\varphi: A \rightarrow M$ and $\widetilde{M}$ and $\widetilde{A}$ and $\tilde{\varphi}: \widetilde{A} \rightarrow \widetilde{M}$, are all defined over $\mathbb{Q}(\zeta)$, the field of $N$ th roots of unity. Therefore $\operatorname{Gal}(\mathbb{Q} / \mathbb{Q}(\zeta))$ acts on the étale cohomology $H_{\text {et }}^{2 d}\left(\tilde{A} \otimes_{\mathbb{Q}(\zeta)} \overline{\mathbb{Q}}, \mathbb{Q}_{l}(d)\right)$, via its natural action on the second factor of $\tilde{A} \otimes_{\mathbb{Q}(\zeta)} \overline{\mathbb{Q}}$ twisted by the action on $\mathbb{Q}_{l}(d)$; and the set of Tate cycles $T^{2 d}(\tilde{A}, \overline{\mathbb{Q}}) \subset H_{\mathrm{et}}^{2 d}\left(\tilde{A} \otimes_{\mathbb{Q}(\zeta)} \overline{\mathbb{Q}}, \mathbb{Q}_{l}(d)\right)$ consists of those classes which are fixed by some open subgroup of $\operatorname{Gal}(\mathbb{Q} / \mathbb{Q}(\zeta))$. Then it is easy to see that every algebraic cycle in $H_{\mathrm{et}}^{2 d}\left(\tilde{A} \otimes_{\mathbb{Q}(\zeta)} \overline{\mathbb{Q}}, \mathbb{Q}_{l}(d)\right)$ is a Tate cycle, and what Tate conjectured (for more general varieties than just $\tilde{A}$ ) is that the converse is also true.

Theorem 5. $T^{2 d}(\tilde{A}, \overline{\mathbb{Q}})$ is spanned by algebraic cycles.

Proof. The main theorem of $p$-adic Hodge theory [F, FM] tells us that if $K$ is a $p$-adic field and $X$ is a smooth proper $K$-scheme, then there is a natural isomorphism

$$
H_{\mathrm{et}}^{m}\left(X \otimes_{K} \bar{K}, \mathbb{Z}_{p}\right) \otimes_{\mathbb{Z}_{p}} \bar{K}^{\wedge} \cong \bigoplus_{a+b=m} H^{a}\left(X, \Omega_{X / K}^{b}\right) \otimes_{K} \bar{K}^{\wedge}(-b),
$$

where the - indicates $p$-adic completion. Then by embedding $\mathbb{Q}(\zeta)$ into some $p$-adic completion, it follows from the comparison and proper base change theorems $[\mathrm{Ml}]$, that

$$
\operatorname{dim}_{\mathbb{Q}_{l}} T^{2 d}(\tilde{A}, \overline{\mathbb{Q}}) \leq \operatorname{dim}_{\mathbb{C}} H^{d, d}(\widetilde{A})
$$

this argument is due to Faltings. Since every algebraic cycle determines a Tate cycle, the result now follows from Theorem 2.

Remark. Of course the same argument verifies the Tate conjecture for and variety for which Theorem 2 is true, in particular for the compact analogue of $\tilde{A}$ considered in [HK, Go2], as well as for projective spaces, Grassmannians and flag varieties.

\section{Proof of Theorem A}

A. In addition to being determined by its Hodge filtration or Hodge decomposition, a rational Hodge structure of weight $m$ may also be defined on a rational vector space $V$ by a homomorphism of real algebraic groups $\alpha: R_{\mathbb{C} / \mathbb{R}} \mathbb{G}_{m / \mathbb{C}} \rightarrow$ $\mathrm{GL}(V, \mathbb{R})$ such that the composition of $\alpha$ with the natural inclusion $\mathbb{G}_{m / \mathbb{R}} \rightarrow$ $R_{\mathbb{C} / \mathbb{R}} \mathbb{G}_{m / \mathbb{C}}$ is given by $x \mapsto x^{m} \cdot 1$, or, if the weight $m$ is given, by a homomorphism $\beta: \mathrm{U}(1) \rightarrow \mathrm{GL}(V, \mathbb{R})$ of real algebraic groups all of whose weights are congruent to $m$ mod $2[\mathrm{Mm} 1, \mathrm{D} 4]$. Using this description of a rational Hodge structure, we may define the special Mumford-Tate group $\operatorname{SMT}(V)$, also called the Hodge group of $V$, as the smallest algebraic subgroup of $\mathrm{GL}(V)$ defined over $\mathbb{Q}$ which contains $\beta(\mathrm{U}(1))$; similarly the Mumford-Tate group $\operatorname{MT}(V)$ is the smallest algebraic subgroup of $\mathrm{GL}(V)$ defined over $\mathbb{Q}$ such that $\operatorname{MT}(V, \mathbb{C})$ contains $\alpha\left(R_{\mathbb{C} / \mathbb{R}} \mathbb{G}_{m / \mathbb{C}}(\mathbb{C})\right)$ [Mm1, D4]. Both groups are connected, and reductive when $V$ is polarizable [D4], which will be the case for all the Hodge structures we consider below. 
If $V$ is a rational Hodge structure of weight $m$, then its $k$ th tensor power $V^{\otimes k}:=V \otimes \cdots \otimes V \quad(k$ factors $)$ is a rational Hodge structure of weight $\mathrm{km}$ on which $\operatorname{SMT}(V)$ acts diagonally. In fact, $\operatorname{SMT}(V)$ may be characterized as the largest subgroup of $\mathrm{GL}(V)$ which fixes all the Hodge vectors, i.e. the rational vectors of Hodge type $(m k / 2, m k / 2)$, in $V^{\otimes k}$ for all $k$ [Mm1, D4]. In particular, $\operatorname{SMT}\left(V^{\otimes k}\right)=\operatorname{SMT}(V)$.

Proposition 1. Let $V$ be a polarizable Hodge structure. Then the rational $\operatorname{SMT}(V)$-invariant subspaces of $V^{\otimes k}$ are precisely the rational sub-Hodge structures.

Proof. If $W$ is a rational sub-Hodge structure of $V^{\otimes k}$, then the map $\beta_{W}$ : $\mathrm{U}(1) \rightarrow \mathrm{GL}(W, \mathbb{R})$ is induced by $\beta^{\otimes k}: \mathrm{U}(1) \rightarrow \mathrm{GL}\left(V^{\otimes k}, \mathbb{R}\right)$. Therefore $W \subseteq V^{\otimes k}$ is an $\operatorname{SMT}(V)$-invariant subspace. Conversely, if $W \subseteq V^{\otimes k}$ is invariant under $\operatorname{SMT}(V)$, then it is a rational sub-Hodge structure via $\mathrm{U}(1) \rightarrow$ $\operatorname{SMT}(V, \mathbb{R}) \rightarrow \mathrm{GL}(W, \mathbb{R})$.

In particular, a rational Hodge structure is irreducible if and only if it is irreducible as a representation of $\operatorname{SMT}(V)$.

B. Recall that the only irreducible rational finite-dimensional representations of $\mathrm{SL}_{2}$ (over a field of characteristic 0 ) are the symmetric tensor representations $\left(\rho_{\nu}, V_{\nu}\right)$ of degree $\nu$ and dimension $\nu+1$, for $\nu \geq 0$, so that $\rho_{0}$ is the trivial represention and $\rho_{1}$ is the identity represention of $\mathrm{SL}_{2}$ on a 2-dimensional space [W]. Moreover, since $\mathrm{SL}_{2}$ is semisimple, any finite-dimensional representation $W$ decomposes into a direct sum of isotypic components

$$
W=\bigoplus_{\nu \geq 0} W\left[\rho_{\nu}\right]
$$

with $W\left[\rho_{\nu}\right]$ isomorphic, as an $\mathrm{SL}_{2}$-module, to the direct sum of some number of copies of $V_{\nu}$. Let $k V_{1}:=V_{1} \oplus V_{1} \oplus \cdots \oplus V_{1}$ ( $k$ factors).

Lemma 1. In the Grassmann algebra $\Lambda^{*}\left(k V_{1}\right)$ the isotypic component $\Lambda^{m}\left(k V_{1}\right)\left[\rho_{\nu}\right]$ of $\Lambda^{m}\left(k V_{1}\right)$ is spanned by the product of $\Lambda^{\nu}\left(k V_{1}\right)\left[\rho_{\nu}\right]$ with $\wedge^{m-\nu}\left(k V_{1}\right)\left[\rho_{0}\right]$.

Proof. It suffices to check that the highest weight vectors of $\bigwedge^{m}\left(k V_{1}\right)$ are products of the highest weight vectors in $\Lambda^{m-\nu}\left(k V_{1}\right)\left[\rho_{0}\right]$ with those of $\Lambda^{\nu}\left(k V_{1}\right)\left[\rho_{\nu}\right]$; see $[\mathrm{Go} 2,2.2]$ for more details.

Moreover, it follows from the Cauchy decomposition formula and the JacobiTrudy formula that the multiplicity of $\rho_{\nu}$ in $\bigwedge^{m}\left(k V_{1}\right)$ is

$$
m(k, m, \nu)=\left(\begin{array}{c}
k \\
(m+\nu) / 2
\end{array}\right)\left(\begin{array}{c}
k \\
(m-\nu) / 2
\end{array}\right)-\left(\begin{array}{c}
k \\
(m+\nu+2) / 2
\end{array}\right)\left(\begin{array}{c}
k \\
(m-\nu-2) / 2
\end{array}\right) ;
$$

[L, (1.7.3), McD, (I.3.5)] (see also [K1, KS]).

C. Proof of Theorem A. Since

$$
H^{m}\left(E^{k}, \mathbb{Q}\right) \simeq \bigwedge^{*}\left(H^{1}(E, \mathbb{Q}) \oplus \cdots \oplus H^{1}(E, \mathbb{Q})\right) \quad(k \text { terms })
$$

(noncanonically), it follows from the remarks preceding Proposition 1 that

$$
\operatorname{SMT}\left(H^{m}\left(E^{k}, \mathbb{Q}\right)\right)=\operatorname{SMT}\left(H^{1}(E, \mathbb{Q})\right)=: \operatorname{SMT}(E) .
$$

Furthermore, since $E$ does not have complex multiplication, we may identify $\operatorname{SMT}(E)$ with $\mathrm{SL}_{2}(\mathbb{Q})$ and its action on $H^{1}(E, \mathbb{Q})$ with $\left(\rho_{1}, V_{1}\right)[\mathrm{Mm} 2]$, 
consequently also identifying $H^{m}\left(E^{k}, \mathbb{Q}\right)$ with $\bigwedge^{m}\left(k V_{1}\right)$. Now Theorem $\mathrm{A}$ will follow from Proposition 1 and Lemma 1 if we know two facts: first, that $H^{m}\left(E^{k}, \mathbb{Q}\right)\left[\rho_{0}\right]$ is spanned by products of divisor classes, and second, that the irreducible $\operatorname{SMT}(E)$-constituents of $H^{\nu}\left(E^{k}, \mathbb{Q}\right)\left[\rho_{\nu}\right]$ are of type $\{(\nu, 0), \ldots$, $(0, \nu)\}$. The first of these facts is equivalent to the usual Hodge conjecture for $E^{k}$, which is known [Mt1, Mt2], or may be proved by the same argument as in $[\mathrm{K} 2, \S \mathrm{F}]$. As for the second statement, it is readily checked that any $\operatorname{SMT}(E)$ irreducible constituent of $H^{\nu}\left(E^{k}, \mathbb{C}\right)\left[\rho_{\nu}\right]$ is isomorphic to the $\nu$ th symmetric tensor power of the Hodge structure $H^{1}(E, \mathbb{C})$ of type $\{(1,0),(0,1)\}$.

\section{Proof of Theorem B}

There are three distinct aspects to the proof of Theorem B: first, the decomposition of $H^{*}(\widetilde{A}, \mathbb{Q})$ as a rational Hodge structure; second, showing that there are algebraic cycles where there are supposed to be, as described in part (i); and third, identifying the Hodge structure, and in particular the Hodge type, of the remaining sub-Hodge structures.

A. Let $h^{*}: H^{*}(\tilde{A}, \mathbb{Q}) \rightarrow H^{*}(A, \mathbb{Q})$ denote the restriction map, and let $\widetilde{H}^{*}(A, \mathbb{Q})$ denote the image of $h^{*}$. Then $\widetilde{H}^{*}(A, \mathbb{Q})$ consists of the pure part in each degree of $H^{*}(A, \mathbb{Q})$, in the sense of [D3], and is therefore independent of the choice of smooth completion $\tilde{A}$. Moreover, since the category of rational Hodge structures is semisimple [D3], and $\operatorname{ker}\left[h^{*}\right]$ is a sub-Hodge structure of $H^{*}(\widetilde{A}, \mathbb{Q})$, we have

$$
H^{*}(\tilde{A}, \mathbb{Q}) \cong \operatorname{ker}\left[h^{*}\right] \oplus \widetilde{H}^{*}(A, \mathbb{Q})
$$

as a rational Hodge structure.

Remark. We will see that there is a natural realization of $\widetilde{H}^{*}(A, \mathbb{Q})$ inside $H^{*}(\widetilde{A}, \mathbb{Q})$ via which $(3.1)$ becomes an equality. Also, Jannsen $[\mathrm{J}]$ has shown that $\operatorname{ker}\left[h^{*}\right]$ and $\operatorname{Im}\left[h^{*}\right]$ are absolute Hodge cycle submotives of $H^{*}(\widetilde{A})$, meaning that $H^{*}(\tilde{A}, \mathbb{Q})$ can be split as in (3.1) using the morphisms associated to absolute Hodge cycles, from which it also follows that there is a realization of $\widetilde{H}^{*}(A, \mathbb{Q})$ as a sub-Hodge structure of $H^{*}(\widetilde{A}, \mathbb{Q})$.

Next we observe that any decomposition of $H^{*}(A, \mathbb{Q})$ as a (possibly mixed) Hodge structure will induce a corresponding decomposition of $\widetilde{H}^{*}(A, \mathbb{Q})$ by restriction. Let $\boldsymbol{\Theta}_{n}$ denote the endomorphism of $A$ induced by multiplication by $n$ in the fiber, for any integer $n>1$, and let $H^{\langle a, b\rangle}(A, \mathbb{Q})$ denote the $n^{b}$-eigenspace of $\boldsymbol{\Theta}_{n}^{*}$ in $H^{a+b}(A, \mathbb{Q})$. Then

$$
H^{m}(A, \mathbb{Q})=H^{\langle 0, m\rangle}(A, \mathbb{Q}) \oplus H^{\langle 1, m-1\rangle}(A, \mathbb{Q}) .
$$

This follows from the observations that since $\varphi: A \rightarrow M$ is proper and smooth, its Leray spectral sequence $E_{r}(\varphi) \Rightarrow H^{*}(A, \mathbb{Q})$ degenerates at the $E_{2}$-term [GH, 3.5], where $E_{2}^{a, b}=H^{a}\left(M, R^{b} \varphi_{*} \mathbb{Q}\right)$, and that $\Theta_{n}^{*}$ commutes with the $d_{2}$ differentials ("Lieberman's trick"), from which one easily finds that

$$
H^{\langle a, b\rangle}(A, \mathbb{Q}) \simeq H^{a}\left(M, R^{b} \varphi_{*} \mathbb{Q}\right) ;
$$

this may be compared with [Ha, $\mathrm{HK}, \mathrm{K} 1-3, \mathrm{Z}]$ with the difference that in the present case $H^{2}\left(M, R^{b} \varphi_{*} \mathbb{Q}\right)=0$ since $M$ is not compact. In particular, as 
the eigenspace of an algebraic morphism, $H^{\langle a, b\rangle}(A, \mathbb{Q})$ is a sub-(mixed) Hodge structure of $H^{m}(A, \mathbb{Q})$, and (3.2) is a decomposition of (mixed) Hodge structures.

Now, since $R^{b} \varphi_{*} \mathbb{Q}$ is the locally constant sheaf on $M$ associated to the action of $\pi_{1}(M) \simeq \Gamma(N) \subset \mathrm{SL}_{2}(\mathbb{Q})$ on $H^{b}\left(A_{\eta}, \mathbb{Q}\right)$, it further decomposes into subsheaves according to the $\mathrm{SL}_{2}$-isotypic decomposition of $H^{b}\left(A_{\eta}, \mathbb{Q}\right) \simeq$ $\Lambda^{b}\left(k V_{1}\right)$ (as in $\left.\S 2\right)$,

$$
R^{b} \varphi_{*} \mathbb{Q}=\bigoplus_{\nu} R^{b} \varphi_{*} \mathbb{Q}\left[\rho_{\nu}\right],
$$

where $R^{b} \varphi_{*} \mathbb{Q}\left[\rho_{\nu}\right]$ is isomorphic to a direct sum of $m(k, b, \nu)$ copies of $\mathscr{V}_{\nu}$, the local coefficient system on $M$ associated to the irreducible representation $\left(\rho_{\nu}, V_{\nu}\right)$. Let $H^{\langle a, b\rangle}\left(A, \mathbb{Q} ;\left[\rho_{\nu}\right]\right)$ denote the subspace of $H^{\langle a, b\rangle}(A, \mathbb{Q})$ isomorphic to $H^{a}\left(M, R^{b} \varphi_{*} \mathbb{Q}\left[\rho_{\nu}\right]\right)$, and let

$$
\widetilde{H}^{\langle a, b\rangle}\left(A, \mathbb{Q} ;\left[\rho_{\nu}\right]\right):=\widetilde{H}^{a+b}(A, \mathbb{Q}) \cap H^{\langle a, b\rangle}\left(A, \mathbb{Q} ;\left[\rho_{\nu}\right]\right) .
$$

Then the decomposition of $H^{m}(\tilde{A}, \mathbb{Q})$ that we seek is described in the following lemma.

Lemma 2. As a rational Hodge structure,

$$
\begin{array}{r}
H^{m}(\tilde{A}, \mathbb{Q}) \cong \operatorname{ker}\left[h^{*}: H^{m}(\tilde{A}, \mathbb{Q}) \rightarrow H^{m}(A, \mathbb{Q})\right] \oplus \tilde{H}^{\langle 0, m\rangle}(A, \mathbb{Q}) \\
\oplus \bigoplus_{0 \leq \nu \leq m-1} \tilde{H}^{\langle 0, m-\nu-1\rangle}(A, \mathbb{Q}) \otimes \tilde{H}^{\langle 1, \nu\rangle}\left(A, \mathbb{Q} ;\left[\rho_{\nu}\right]\right),
\end{array}
$$

where the tensor product is realized by the cup product in $H^{*}(A, \mathbb{Q})$. Moreover, $\widetilde{H}^{\langle 0, m\rangle}(A, \mathbb{Q})=\widetilde{H}^{\langle 0, m\rangle}\left(A, \mathbb{Q} ;\left[\rho_{0}\right]\right)$, while $\widetilde{H}^{\langle 1, \nu\rangle}\left(A, \mathbb{Q} ;\left[\rho_{\nu}\right]\right)$ is isomorphic to the direct sum of $\left(\begin{array}{l}k \\ \nu\end{array}\right)$ copies of $H^{1}\left(\widetilde{M}, j_{*} \mathscr{V}_{\nu}\right)$.

Proof. From (3.2) it immediately follows that

$$
\widetilde{H}^{m}(A, \mathbb{Q})=\widetilde{H}^{\langle 0, m\rangle}(A, \mathbb{Q}) \oplus \widetilde{H}^{\langle 1, m-1\rangle}(A, \mathbb{Q})
$$

is a Hodge structure decomposition. Furthermore, $H^{\langle 0, m\rangle}\left(A, \mathbb{Q} ;\left[\rho_{\nu}\right]\right)$ vanishes unless $m$ is even and $\nu=0$, which proves that

$$
\widetilde{H}^{\langle 0, m\rangle}(A, \mathbb{Q})=\widetilde{H}^{\langle 0, m\rangle}\left(A, \mathbb{Q} ;\left[\rho_{0}\right]\right)
$$

as claimed.

From (3.3) and (3.4) it is clear that

$$
H^{\langle 1, b\rangle}(A, \mathbb{Q})=\bigoplus_{0 \leq \nu \leq b} H^{\langle 1, b\rangle}\left(A, \mathbb{Q} ;\left[\rho_{\nu}\right]\right) .
$$

To see that this is a Hodge structure decomposition, the methods of [Gol] may be applied. There it is shown that one may choose any $\mathrm{SL}_{2}$-submodule of $\Lambda^{b}\left(k V_{1}\right)$, say $U$, and therefore any (locally constant) subsheaf $\mathscr{U}$ of $R^{b} \varphi_{*} \mathbb{Q}$, and construct an algebraic cycle in $A$ which induces a projection from $H^{1+b}(A, \mathbb{Q})$ to the subspace of $H^{\langle 1, b\rangle}(A, \mathbb{Q})$ identified by $(3.3)$ with $H^{1}(M, \mathscr{U})$. Thus

$$
\widetilde{H}^{\langle 1, b\rangle}(A, \mathbb{Q})=\bigoplus_{0 \leq \nu \leq b} \widetilde{H}^{\langle 1, b\rangle}\left(A, \mathbb{Q} ;\left[\rho_{\nu}\right]\right)
$$

as a rational Hodge structure. 
Now from Lemma 1 and the identifications preceding (3.4) it follows that

$$
R^{b} \varphi_{*} \mathbb{Q}\left[\rho_{\nu}\right]=R^{\nu} \varphi_{*} \mathbb{Q}\left[\rho_{\nu}\right] \otimes R^{b-\nu} \varphi_{*} \mathbb{Q}\left[\rho_{0}\right] .
$$

Therefore from the compatibility of the cup product on $H^{*}(A, \mathbb{Q})$ with the cup products along the base and the fiber (here we are using that the Leray spectral sequence $E_{r}(\varphi) \Rightarrow H^{*}(A, \mathbb{Q})$ degenerates at the $E_{2}$-term $)$ [Wh, 13.8] we may conclude that

$$
\widetilde{H}^{\langle 1, b\rangle}\left(A, \mathbb{Q} ;\left[\rho_{\nu}\right]\right) \cong \widetilde{H}^{\langle 1, \nu\rangle}\left(A, \mathbb{Q} ;\left[\rho_{\nu}\right]\right) \otimes \widetilde{H}^{\langle 0, b-\nu\rangle}\left(A, \mathbb{Q} ;\left[\rho_{0}\right]\right),
$$

which proves the first part of the lemma.

Finally, it remains to show that

$$
\widetilde{H}^{\langle 1, \nu\rangle}\left(A, \mathbb{Q} ;\left[\rho_{\nu}\right]\right) \simeq H^{1}\left(\widetilde{M}, j_{*} R^{\nu} \varphi_{*} \mathbb{Q}\left[\rho_{\nu}\right]\right) .
$$

However, the Leray spectral sequence $E_{r}(\tilde{\varphi}) \Rightarrow H^{*}(\tilde{A}, \mathbb{Q})$ degenerates at the $E_{2}$-term $[\mathrm{Z}, \S 15]$, and $h^{*}$ induces a map of spectrai sequences $E_{r}^{a, b}(\tilde{\varphi}) \rightarrow$ $E_{r}^{a, b}(\varphi)$. Therefore,

$$
\widetilde{H}^{\langle 1, \nu\rangle}(A, \mathbb{Q}) \simeq \operatorname{Im}\left[H^{1}\left(\widetilde{M}, R^{\nu} \tilde{\varphi}_{*} \mathbb{Q}\right) \rightarrow H^{1}\left(M, R^{\nu} \varphi_{*} \mathbb{Q}\right)\right],
$$

and likewise for the $\rho_{\nu}$-constituents. On the other hand, from the invariant cycle theorem [D3, C] it follows that $H^{1}\left(\widetilde{M}, R^{\nu} \tilde{\varphi}_{*} \mathbb{Q}\right)$ maps surjectively onto $H^{1}\left(\widetilde{M}, j_{*} R^{\nu} \varphi_{*} \mathbb{Q}\right)$, while an examination of the Leray spectral sequence associated to $j: M \hookrightarrow \widetilde{M}$ shows that there is an injection of $H^{1}\left(\widetilde{M}, j_{*} R^{\nu} \varphi_{*} \mathbb{Q}\right)$ into $H^{1}\left(M, R^{\nu} \varphi_{*} \mathbb{Q}\left[\rho_{\nu}\right]\right)$. Since the same surjectivity will hold true when the coefficients are replaced by their $\rho_{\nu}$-constituents, this proves 3.5 and the lemma.

B. Next we look at the algebraic cycles in $H^{*}(\tilde{A}, \mathbb{Q})$.

Lemma 3. There is a natural realization of $\widetilde{H}^{\langle 0, *\rangle}(A, \mathbb{Q})$ in $H^{*}(\tilde{A}, \mathbb{Q})$ which is generated by the classes of divisors on $\tilde{A}$ which are the closures in $\tilde{A}$ of divisors on $A_{\eta}$.

Proof. To begin we view $\widetilde{H}^{\langle 0, b\rangle}(A, \mathbb{Q})$ as a subalgebra of $H^{b}(A, \mathbb{Q})$. From (3.3), Lemma 2 and the proof of Theorem A,

$$
\widetilde{H}^{\langle 0, b\rangle}(A, \mathbb{Q}) \simeq H^{0}\left(\widetilde{M}, j_{*} R^{b} \varphi_{*} \mathbb{Q}\right) \simeq H^{b}\left(A_{\eta}, \mathbb{Q}\right)\left[\rho_{0}\right]=A H^{b}\left(A_{\eta}, \mathbb{Q}\right),
$$

where these isomorphisms are fully compatible with the cup product algebra structures of these cohomology rings. On the other hand, for each algebraic cycle on $A_{\eta}$ we may take its closure with respect to the generic point $\eta$ of $\widetilde{M}$ to obtain an algebraic cycle on $\widetilde{A}$ which determines a class in $\widetilde{H}^{\langle 0, *\rangle}(A, \mathbb{Q})$. And since $A H^{*}\left(A_{\eta}, \mathbb{Q}\right)$ is generated by divisors and the closure of a divisor on $A_{\eta}$ is a divisor on $\tilde{A}$, the result follows.

Lemma 4. $\operatorname{ker}\left[h^{*}\right]$ consists of algebraic cycles.

Proof. Let $\sigma: \widetilde{A^{\infty}} \rightarrow A^{\infty}$ be a resolution of singularities, and let $\psi: \widetilde{A^{\infty}} \rightarrow \tilde{A}$ be the composition of $\sigma$ with the inclusion $A^{\infty} \hookrightarrow \widetilde{A}$. Then for each $m$

$$
\operatorname{ker}\left[h^{*}\right]=\operatorname{Im}\left[\psi_{*}: H^{m-2}\left(\widetilde{A^{\infty}}, \mathbb{Q}\right) \rightarrow H^{m}(\tilde{A}, \mathbb{Q})\right]
$$


[D3, 8.2.8], so it suffices to check that $H^{*}\left(\widetilde{A^{\infty}}, \mathbb{Q}\right)$ is spanned by algebraic cycles. However, $\widetilde{A^{\infty}}$ consists of the irreducible components of $A^{\infty}$, since $A^{\infty}$ is a divisor with normal crossings as a subvariety of $\widetilde{A}$. And it follows from the construction of $\bar{A}$ and $\widetilde{A}$ (1D, also see [Š1]) that all these components belong to the category of algebraic varieties generated by rational curves and the operations of direct product and blowing-up over smooth centers. Since these operations preserve the property that the cohomology ring is spanned by algebraic cycles, the lemma follows.

C. Implicit in the next lemma is that there are no other algebraic cycles in $H^{*}(\widetilde{A}, \mathbb{Q})$ than those just described above.

Lemma 5. $\widetilde{H}^{\langle 1, \nu\rangle}\left(A, \mathbb{Q} ;\left[\rho_{\nu}\right]\right)$ is a rational Hodge structure of type $\{(\nu+1,0)$, $(0, \nu+1)\}$ with $\mathbb{Q}$-rank $\frac{1}{12}\left(\begin{array}{l}k \\ \nu\end{array}\right)(\nu N+N+6) N^{2} \prod_{p \mid N}\left(1-p^{-2}\right)$.

Proof. After the last part of Lemma 2, it suffices to prove that the subspace $H^{1}\left(\widetilde{M}, j_{*} \mathscr{V}_{\nu}\right) \subset \widetilde{H}^{\langle 1, \nu\rangle}(A, \mathbb{Q})$, is a rational Hodge structure of type $\{(\nu+$ $1,0),(0, \nu+1)\}$ with $\mathbb{Q}$-rank $\frac{1}{12}(\nu N+N+6) N^{2} \prod_{p \mid N}\left(1-p^{-2}\right)$. However, following [Š2] (see also [Z, §12]), we observe that by the Eichler-Shimura isomorphism

$$
S_{\nu+2}(\Gamma(N)) \oplus \overline{S_{\nu+2}(\Gamma(N))} \stackrel{\sim}{\rightarrow} H^{1}\left(\widetilde{M}, j_{*} \mathscr{V}_{\nu}(\mathbb{C})\right),
$$

where $S_{\nu+2}(\Gamma(N))$ denotes the space of holomorphic cusp forms of weight $\nu+2$ for $\Gamma(N)\left[\mathrm{Sm} 1, \mathrm{Sm} 2\right.$, V]. Moreover, if we represent $A$ as $\Gamma(N) \ltimes \mathbb{Z}^{2 k} \backslash \mathfrak{H} \times \mathbb{C}^{k}$, then $f \in S_{\nu+2}(\Gamma(N))$ determines a differential form $f(\tau) d \tau d z_{i_{1}} \cdots d z_{i_{\nu}}$ of type $(\nu+1,0)$ on $A$ which extends to $\tilde{A}$ because $f$ is cuspidal [Š2]. Since it is readily deduced from $[\mathrm{Sm} 1, \S 2.6]$ and $[\mathrm{Sb}$, Chapter IV] that

$$
\operatorname{dim}_{\mathbb{C}} S_{\nu+2}(\Gamma(N))=\frac{1}{24}(\nu N+N+6) N^{2} \prod_{p \mid N}\left(1-p^{-2}\right),
$$

the lemma follows.

D. To complete the proof of Theorem B, it follows from the proof of Lemma 5 above that there is an explicit realization of $\widetilde{H}^{\langle 1, \nu\rangle}\left(A, \mathbb{Q} ;\left[\rho_{\nu}\right]\right)$ inside $H^{\nu+1}(\tilde{A}, \mathbb{Q})$; see [SI]. With Lemmas 4 and 3 , this implies that the decomposition of the Hodge structure $H^{m}(\widetilde{A}, \mathbb{Q})$ given by Lemma 2 corresponds to a genuine decomposition of $H^{m}(\tilde{A}, \mathbb{Q})$ as cohomology group. Moreover, $\operatorname{ker}\left[h^{*}\right]$ and the preimage of $\widetilde{H}^{\langle 0, *\rangle}(A, \mathbb{Q})$ described in Lemma 3 consist of algebraic cycles, while their complement in $H^{*}(\widetilde{A}, \mathbb{Q})$ whose Hodge structure is described in Lemma 5 cannot contain any algebraic cycles; part (i) of the theorem follows. Part (ii) follows directly from Lemmas 2, 3 and 5.

\section{ACKNOWLEDGMENTS}

The author thanks D. Blasius, S. Katz, K. Murty, D. Ramakrishnan, and C. Schoen for many helpful and friendly conversations, and the Mathematical Sciences Research Institute, Berkeley, for its generous hospitality during the period when these ideas were being developed. 


\section{REFERENCES}

[AMRT] A. Ash, D. Mumford, M. Rapoport and Y. Tai, Smooth compactifications of locally symmetric spaces, Math. Sci. Press, Brookline, Mass., 1975.

[BO] S. Bloch and A. Ogus, Gersten's conjecture and the homology of schemes, Ann. Sci. Ecole Norm. Sup. 7 (1974), 181-202.

[C] C. H. Clemens, Degeneration of Kähler manifolds, Duke Math. J. 44 (1977), 215-290.

[D1] P. Deligne, Formes modulaires et représentations l-adiques, Séminaire Bourbaki, Lecture Notes in Math., vol 179, Springer, New York, 1971, pp. 139-172.

[D2] _ Travaux de Shimura, Séminaire Bourbaki, vol. 1970/71, Exposés 382-399, Lecture Notes in Math., vol. 244, Springer, New York and Berlin, 1971, pp. 123-165.

[D3] _ Théorie de Hodge. II, III, Publ. Math. Inst. Hautes Etudes Sci. 40 (1971), 5-58; 44 (1974), 5-77.

[D4] Hodge cycles on abelian varieties (Notes by J. S. Milne), Hodge Cycles, Motives, and Shimura Varieties, Lecture Notes in Math., vol. 900, Springer, New York, 1982, pp. 9-100.

[F] G. Faltings, p-adic Hodge theory, J. Amer. Math. Soc. 1 (1988), 255-299.

[FM] J.-M. Fontaine and W. Messing, p-adic periods and p-adic étale cohomology, preprint.

[Go1] B. B. Gordon, Algebraically defined subspaces in the cohomology of a Kuga fiber variety, Pacific J. Math. 131 (1988), 261-276.

[Go2] B. B. Gordon, Topological and algebraic cycles in Kuga-Shimura varieties, Math. Ann. 279 (1988), 395-402.

[Gf1] P. A. Griffiths, Some results on algebraic cycles on algebraic manifolds, Algebraic Geometry, Bombay 1968, Oxford Univ. Press, New York and Oxford, 1969, pp. 93-191.

[Gf2] - Some transcendental methods in the study of algebraic cycles, Several Complex Variables II, Maryland 1980, Lecture Notes in Math., vol. 185, Springer, New York,, 1971, pp. 1-46.

[GH] P. Griffiths and J. Harris, Principles of algebraic geometry, Wiley, New York, 1978.

[Gr1] A. Grothendieck, Hodge's general conjecture is false for trivial reasons, Topology 8 (1969), 299-303.

[Gr2] L Le groupe de Brauer III: Exemples et compléments, Dix exposé sur la cohomologie des schémas, Adv. Stud. in Pure Math., vol. 3, North-Holland, Amsterdam, 1968, pp. 88-188.

[Ha] R. Hall, On the cohomology of Kuga's fiber variety, Pacific J. Math. 75 (1975), 411-422.

[HK] R. Hall and M. Kuga, Algebraic cycles in a fiber variety, Sci. Papers College Gen. Ed. Univ. Tokyo 25 (1975), 1-6.

[H] W. V. D. Hodge, The topological invariants of algebraic varieties, Proc. Inernat. Congr. Math. (1950), 182-182.

[J] U. Jannsen, Mixed motives, preprint.

[Ko] K. Kodaira, On compact complex analytic surfaces. I, II, III, Ann. of Math. 71 (1960), 111-152; 77 (1963), 563-626; 78 (1963), 1-40.

[K1] M. Kuga, Fibre varieties over a symmetric space whose fibres are abelian varieties, vols. I and II, Univ. of Chicago, Chicago, Ill., 1964.

[K2] - Fibre varieties over symmetric space whose fibres are abelian varieties, Proc. of the U.S.Japan Seminar in Differential Geometry, Kyoto, Japan, 1965, Nippon Hyoronsha, 1966, pp. 72-81.

[K3] - Algebraic cycles in gtfabv, J. Fac. Sci. Univ. Tokyo Sect. IA Math. 29 (1982), 13-29.

[KL] M. Kuga and J. V. Leahy, Shimura's abelian varieties as Weil's higher Jacobian varieties, J. Fac. Sci. Univ. Tokyo Sect. I 16 (1969), 229-253.

[KS] M. Kuga and G. Shimura, On the zeta function of a fiber variety whose fibres are abelian varieties, Ann. of Math. 82 (1965), 487-539.

[L] A. Lascoux, Syzygies des variétés déterminantales, Adv. in Math. 30 (1978), 202-237.

[McD] I. G. MacDonald, Symmetric functions and Hall polynomials, Clarendon Press, Oxford, 1979. 
[Ml] J. S. Milne, Etale cohomology, Princeton Univ. Press, Princeton, N. J., 1980.

[Mm1] D. Mumford, Families of abelian varieties, Algebraic Groups and Discontinuous Subgroups, Proc. Sympos. Pure Math., vol. 9, Amer. Math. Soc, Providence, R. I., 1966, pp. 347-351.

[Mm2] _ A note on Shimura's paper "Discontinuous groups and abelian varieties", Math. Ann. 181 (1969), 345-351.

[Mr] J. P. Murre, Abel-Jacobi equivalence versus incidence equivalence for algebraic cycles of codimension two, Topology 24 (1985), 361-367.

[Mt1] V. K. Murty, Algebraic cycles on abelian varieties, Duke Math. J. 50 (1983), 487-504.

[Mt2] _ Exceptional Hodge classes on certain abelian varieties, Math. Ann. 268 (1984), 197-206.

[Sb] B. Schoeneberg, Elliptic modular functions, Springer-Verlag, New York, Heidelberg and Berlin, 1974.

[S1] A. J. Scholl, Motives for modular forms, preprint.

[Sm1] G. Shimura, Introduction to the arithmetic theory of automorphic forms, Publ. Math. Soc. Japan 11, Princeton Univ. Press, Princeton, N. J., 1971.

[Sm2] _ Sur les intégrales attachées aux formes automorphes, J. Math. Soc. Japan 11 (1959), 291-311.

[Sm3] _ On canonical models of arithmetic quotients of bounded symmetric domains. I, II, Ann. of Math. (2) 91 (1970), 144-222; (2) 92 (1970), 528-549.

[Sol] T. Shioda, What is known about the Hodge conjecture?, Algebraic Varieties and Analytic Varieties, Adv. Stud. in Pure Math. 1, Kinokuniya, Tokyo, 1983, pp. 55-68.

[So2] _ On elliptic modular surfaces, J. Math. Soc. Japan 24 (1972), 20-59.

[Š1] V. V. Šokurov, Holomorphic differential forms of higher degree on Kuga's modular varieties, Math. USSR Sb. 30 (1976), 1199-142.

[Š2] _ The study of the homology of Kuga varieties, Math USSR Izv. 16 (1981), 399-418.

[T] J. Tate, Algebraic cycles and poles of zeta functions, Arithmetical Algebraic Geometry, Harper and Row, New York, 1965, pp. 93-110.

[V] J.-L. Verdier, Sur les intǵrales attachées aux formes automorphes (d'après Goro Shimura), Séminaire Bourbaki (1961).

[W] H. Weyl, The classical groups, Princeton Univ. Press, Princeton, N. J., 1946.

[Wh] G. W. Whitehead, Elements of homotopy theory, Springer, New York, 1978.

[Z] S. Zucker, Hodge theory with degenerating coefficients: $L_{2}$-cohomology in the Poincaré metric, Ann. of Math. 109 (1979), 415-476.

Department of Mathematics, University of OKLahoma, Norman, OKLahoma 73019

E-mail address: bgordon@nsfuvax.math.uoknor.edu 